\title{
Mild inflammation may switch on again atrial fibrillation after successful electrical cardioversion
}

\author{
Filippo Marzot · Vittorio Pengo
}

Received: 11 May 2009/Accepted: 13 May 2009/Published online: 9 June 2009

(C) SIMI 2009

In recent years, the presence of a low level of systemic inflammation has been shown to be a marker for the onset of atrial fibrillation (AF) and its persistence [1-3]. Laboratory and histological findings support this association, and a relation between the level of inflammation and the arrhythmic burden was demonstrated [1-3]. The paper by Vizzardi et al. [4] in Internal and Emergency Medicine reinforces previous data showing that inflammation plays a role in predicting recurrence of $\mathrm{AF}$ after spontaneous or induced (pharmacologically or with electrical cardioversion) return to sinus rhythm [4-6]. Altogether, this evidence suggests that structural (anatomical, histological and electrophysiological) changes in the atrial tissue induced by an inflammatory state may induce the onset or recurrence of $\mathrm{AF}$.

The paper by Vizzardi et al. [4] deals with the role of plasma high-sensitivity $\mathrm{C}$ reactive protein (CRP) levels in predicting recurrence of AF after successful cardioversion. Patients with structurally healthy heart, without previous rhythm control therapy, undergoing electrical cardioversion for new-onset AF were tested for plasma CRP with an ultrasensitive method. CRP levels were the only variable, which was significantly different between the group of patients who experienced a recurrence of $\mathrm{AF}$ and those who did not in the 6 months following the procedure.

The absence of relationship between factors known to be associated with AF (i.e. hypertension, left atrial

F. Marzot · V. Pengo $(\bowtie)$

Department of Cardiac Thoracic and Vascular Sciences, Clinical

Cardiology, Thrombosis Center, School of Medicine, University

of Padua, via Giustiniani 2, 35128 Padua, Italy

e-mail: vittorio.pengo@unipd.it dimensions, duration of $\mathrm{AF}$ ) and its recurrence after cardioversion in this study is probably due to the type of patients enrolled: all had AF of recent onset, in absence of structural heart disease; patients with severe left atrial dilation, reduced left ventricle ejection fraction, kidney failure or pro-inflammatory conditions were excluded. It is probably in the type of population enrolled that latent systemic inflammation plays a significant role in the pathogenesis of $\mathrm{AF}$, as it does in patients with postoperative $\mathrm{AF}$.

Although this paper presents some limitations, like the sole CRP determination before cardioversion and the inability to detect asymptomatic AF episodes during the follow-up period, the magnitude of difference between patients with AF recurrence and the others calls for a strong predictive value of this inflammation marker. Unfortunately, we do not have a CRP measurement at the time of recurrence but this is obviously linked to the perception of the arrhythmia by the patients. CRP levels were not significantly different between the group of patients who experienced early (i.e. in the first week) recurrence and those who suffered from a late one (i.e. within 6 months). Other inflammatory markers may help to identify patients who are at high risk for early recurrence thus warranting pre-cardioversion rhythm control therapy.

In any case, this method may prove useful in identifying patients in whom a new cardioversion is not warranted.

Other studies may offer further insight on the nature of the relationship between inflammation and $\mathrm{AF}$, and this collective body of work could lay the foundation for trials investigating the role of anti-inflammatory drug therapy in preventing the onset or AF recurrence.

Conflict of interest statement The authors declare that they have no conflict of interest related to the publication of this manuscript. 


\section{References}

1. Chung MK, Martin DO et al (2001) C-reactive protein elevation in patients with atrial arrhythmias inflammatory mechanisms and persistence of atrial fibrillation. Circulation 104:2886-2891

2. Aviles RJ, Martin DO et al (2003) Inflammation as a risk factor for atrial fibrillation. Circulation 108:3006-3010

3. Frustaci A, Chimenti C, Bellocci F, Morgante E, Russo MA, Maseri A (1997) Histological substrate of atrial biopsies in patients with lone atrial fibrillation. Circulation 96:1180-1184

4. Vizzardi E, Nodari S, Zanini G, Manerba A, Frattini S, Madureri A, Dei Cas L (2009) High sensitivity C reactive protein: a predictor for recurrence of atrial fibrillation after successful cardioversion. Int Emerg Med. doi:10.1007/s11739-009-0235-6

5. Hatzinikolaou-Kotsakou E, Tziakas D, Hotidis D et al (2006) Relation of C-reactive protein to the first onset and the recurrence rate in lone atrial fibrillation. Am J Cardiol 97:659-661

6. Malouf JF, Kanagala R, Al Atawi F et al (2005) High sensitivity C-reactive protein. A novel predictor for recurrence of atrial fibrillation after successful cardioversion. J Am Coll Cardiol 46:1284-1287 